Journal of Mathematics and Statistics 6 (2): 100-104, 2010

ISSN 1549-3644

(C) 2010 Science Publications

\title{
Comparison of the Bayesian and Maximum Likelihood Estimation for Weibull Distribution
}

\author{
Al Omari Mohammed Ahmed, Hadeel Salim Al-Kutubi and Noor Akma Ibrahim \\ Department of Mathematics, Faculty of Science, University Putra Malaysia \\ Institute for Mathematical Research, University Putra Malaysia, \\ 43400, UPM, Serdang, Selangor, Malaysia
}

\begin{abstract}
Problem statement: The Weibull distribution has been widely used especially in the modeling of lifetime event data. It provides a statistical model which has a wide variety of applications in many areas, and the main advantage is its ability in the context of lifetime event, to provide reasonably accurate failure analysis and failure forecasts especially with extremely small samples. The conventional maximum likelihood method is the usual way to estimate the parameters of a distribution. Bayesian approach has received much attention and in contention with other estimation methods. In this study we explore and compare the performance of the maximum likelihood estimate with the Bayesian estimate for the Weibull distribution. Approach: The maximum likelihood estimation, Bayesian using Jeffrey prior and the extension of Jeffrey prior information for estimating the parameters of Weibull distribution of life time are presented. We explore the performance of these estimators numerically under varying conditions. Through the simulation study comparison are made on the performance of these estimators with respect to the Mean Square Error (MSE) and Mean Percentage Error (MPE). Results: For all the varying sample size, several specific values of the scale parameter of the Weibull distribution and for the values specify for the extension of Jeffrey prior, the estimators of the maximum likelihood method result in smaller MSE and MPE compared to Bayesian in majority of the cases. Nevertheless in all cases for both methods the MSE and MPE decrease as sample size increases. Conclusion: Based on the results of this simulation study the Bayesian approach used in the estimating of Weibull parameters is found to be not superior compared to the conventional maximum likelihood method with respect to MSE and MPE values.
\end{abstract}

Key words: Extension of Jeffrey prior information, Weibull distribution, Bayes method

\section{INTRODUCTION}

The Weibull distribution is found to be useful in modeling and analyzing life time data in the fields, medicine, biology, engineering sciences and others. Hossain and Zimmer (2003) have discussed some comparative estimation of Weibull parameters using complete and censored samples. Besides, because of its useful applications, an array of method have been proposed for estimating parameters of the Weibull distribution. The most-used methods which are considered to be the traditional methods are maximum likelihood and the moment estimation (Cohen and Whitten, 1982).

The efficiency of the maximum likelihood estimation method makes it popular and the moment estimation method is computationally easy and provides explicit estimators of the parameters. Bayes estimator of three parameters of the Weibull distribution in comparison with the posterior standard deviation estimates counterparts cum numerical example were obtained and given by Sinha and Sloan (1988). They proposed a method based on the primary information with weighted Bayes. Also, using record statistics from Weibull model Bayesian and non-Bayesian approaches, Soliman et al. (2006) carried out the comparison of the estimates. Kantar and Penoolu (2008) did comparative study for the location and scale parameters of the Weibull distribution with a given shape parameter. In this study we propose Bayes with Jeffrey prior and extension of Jeffrey prior information for the Weibull parameters estimation. Soland et al. (1969) introduce Bayesian analysis of the Weibull Process with unknown scale and shape parameters.

The rest of the study is arranged as follows. In Methods, maximum likelihood estimation, Bayes

Corresponding Author: Al Omari Mohammed Ahmed, Institute for Mathematical Research, University Putra Malaysia, 43400, UPM, Serdang, Selangor, Malaysia 
estimator with the Jeffrey prior and the new extension of Jeffrey prior information are presented. In Results, simulation study is discussed and the results are presented and followed by the conclusion

\section{MATERIALS AND METHODS}

Maximum likelihood estimation: We introduce the concept of maximum likelihood estimation with Weibull distribution. We have set of random lifetime $t_{1}, \ldots, t_{n}$ and vectors of unknown parameters $\theta=\left(\theta_{1}, \ldots\right.$, $\left.\theta_{\mathrm{n}}\right)$ and $\mathrm{p}=\left(\mathrm{p}_{1}, \ldots, \mathrm{p}_{\mathrm{n}}\right)$ then Let $(\mathrm{t}, \theta, \mathrm{p})$ is the likelihood function:

$L(t ; \theta, p)=\prod_{i=1}^{n} f\left(t_{i} ; \theta, p\right)$

The ith elements of the score vector with respect to $\theta$ and $\mathrm{p}$ :

$$
\begin{aligned}
& \mathrm{U}_{\mathrm{i}}(\theta)=\frac{\partial \mathrm{L}(\mathrm{t}, \theta, \mathrm{p})}{\partial \theta_{\mathrm{i}}} \\
& \mathrm{U}_{\mathrm{i}}(\mathrm{p})=\frac{\partial \mathrm{L}(\mathrm{t}, \theta, \mathrm{p})}{\partial \mathrm{p}_{\mathrm{i}}}
\end{aligned}
$$

Let $\left(t_{1}, \ldots, t_{n}\right)$ be the set of random lifetime from Weibull distribution with parameters $\theta$ and $p$.

The probability density function of Weibull distribution is given:

$$
f(t ; \theta, p)=\frac{p}{\theta} t^{p-1} \exp \left(-\frac{t^{p}}{\theta}\right)
$$

The likelihood function is:

$$
\begin{aligned}
\mathrm{L}(\mathrm{t} ; \theta, \mathrm{p}) & =\prod_{\mathrm{i}=1}^{\mathrm{n}} \mathrm{f}\left(\mathrm{t}_{\mathrm{i}} ; \theta, \mathrm{p}\right) \\
& =\prod_{\mathrm{i}=1}^{\mathrm{n}} \frac{\mathrm{p}}{\theta} \exp \left(-\frac{\mathrm{t}_{\mathrm{i}}^{\mathrm{p}}}{\theta}\right)
\end{aligned}
$$

The score vectors are:

$$
\begin{aligned}
\mathrm{U}(\theta) & =\frac{\partial \ln \mathrm{L}(\mathrm{t} ; \theta, \mathrm{p})}{\partial \theta} \\
& =-\frac{\mathrm{n}}{\theta}+\frac{\sum_{\mathrm{i}=1}^{\mathrm{n}} \mathrm{t}_{\mathrm{i}}^{\mathrm{p}}}{\theta^{2}}
\end{aligned}
$$

$$
\begin{aligned}
\mathrm{U}(\mathrm{p}) & =\frac{\partial \ln \mathrm{L}(\mathrm{t} ; \theta, \mathrm{p})}{\partial \mathrm{p}} \\
& =-\frac{\mathrm{n}}{\theta}+\sum_{\mathrm{i}=1}^{\mathrm{n}} \ln \left(\mathrm{t}_{\mathrm{i}}\right)-\frac{\sum_{\mathrm{i}=1}^{\mathrm{n}} \mathrm{t}_{\mathrm{i}}^{\mathrm{p}} \ln \left(\mathrm{t}_{\mathrm{i}}\right)}{\theta}
\end{aligned}
$$

Let $U(\theta)$ equal to zero, then the maximum likelihood estimator is:

$$
\hat{\theta}_{M}=\frac{\sum_{i=1}^{n} t_{i}^{p}}{n}
$$

The shape parameter $\mathrm{p}$ is taken to be constant.

Bayes estimation: Let $t_{1}, \ldots, t_{n}$ be a random sample of size $\mathrm{n}$ with distribution function $\mathrm{F}(\mathrm{t}, \theta, \mathrm{p})$ and probability density function $\mathrm{f}(\mathrm{t}, \theta, \mathrm{p})$.

In the Weibull case, we assumed that the probability density function of the lifetime is given by:

$f(t ; \theta, p)=\frac{p}{\theta} t^{p-1} \exp \left(-\frac{t^{p}}{\theta}\right)$

Jeffrey prior information: We find Jeffery prior by taking $\mathrm{g}(\mathrm{q}) \propto \sqrt{\mathrm{I}(\theta)}$, where:

$\mathrm{g}(\theta)=\mathrm{k} \sqrt{\mathrm{I}(\theta)}$ then

$\mathrm{g}(\theta)=\mathrm{k} \frac{\sqrt{\mathrm{n}}}{\theta}$ with $\mathrm{k}$ a constant

We can found Bayes estimator with Jeffrey prior of parametric distribution by using conditional distribution, depend on joint probability density function and marginal probability density function, so the conditional distribution is given by:

$\Pi\left(t_{1}, \ldots ., t_{n} ; \theta, p\right)=\frac{H\left(t_{1}, \ldots ., t_{n} ; \theta, p\right)}{p\left(t_{1}, \ldots ., t_{n}\right)}$

The joint probability density function $\left.f\left(t_{1}, \ldots, t_{n}, \theta, p\right)\right)$ is given by:

$$
\begin{aligned}
H\left(t_{1}, \ldots ., t_{n} ; \theta, p\right) & =\prod_{i=1}^{n} f(t ; \theta, p) g(\theta) \\
& =\frac{k \sqrt{n p^{n}}}{\theta^{n+1}}\left((p-1) \sum_{i=1}^{n} \ln L_{i}-\frac{\sum_{i=1}^{n} t_{i}^{p}}{\theta}\right)
\end{aligned}
$$


The marginal probability density function of $\left(t_{1}, \ldots, t_{n}\right)$ is given by:

$$
\begin{aligned}
\mathrm{p}\left(\mathrm{t}_{1}, \ldots, \mathrm{t}_{\mathrm{n}}\right) & =\int \mathrm{H}\left(\mathrm{t}_{1}, \ldots, \mathrm{t}_{\mathrm{n}}\right) \mathrm{d} \theta \\
& =\frac{\mathrm{k} \sqrt{\mathrm{np} p^{\mathrm{n}}(\mathrm{n}-1) !}}{\left(\sum_{\mathrm{i}=1}^{\mathrm{n}} \mathrm{t}_{\mathrm{i}}^{\mathrm{p}}\right)^{\mathrm{n}}} \exp \left((\mathrm{p}-1) \sum_{\mathrm{i}=1}^{\mathrm{n}} \ln \mathrm{t}_{\mathrm{i}}\right)
\end{aligned}
$$

Then the conditional probability density function of $\theta$ given the data $\left(\mathrm{t}_{1}, \ldots, \mathrm{t}_{\mathrm{n}}\right)$ by:

$$
\begin{aligned}
\Pi\left(\mathrm{t}_{1}, \ldots, \mathrm{t}_{\mathrm{n}} ; \theta, \mathrm{p}\right) & =\frac{\mathrm{H}\left(\mathrm{t}_{1}, \ldots, \mathrm{t}_{\mathrm{n}} ; \theta, \mathrm{p}\right)}{\mathrm{p}\left(\mathrm{t}_{1}, \ldots, \mathrm{t}_{\mathrm{n}}\right)} \\
& =\frac{\left(\sum_{\mathrm{i}=1}^{\mathrm{n}} \mathrm{t}_{\mathrm{i}}^{\mathrm{p}}\right)^{\mathrm{n}}}{\theta^{\mathrm{n}+1}(\mathrm{n}-1) !} \exp \left(-\frac{\sum_{\mathrm{i}=1}^{\mathrm{n}} \mathrm{t}_{\mathrm{i}}^{\mathrm{p}}}{\theta}\right.
\end{aligned}
$$

By using squared error loss function $\ell(\hat{\theta}-\theta)=c(\hat{\theta}-\theta)^{2}$, the Risk function is:

$$
\begin{aligned}
\mathrm{R}(\hat{\theta}-\theta) & =\mathrm{E} \ell(\hat{\theta}-\theta) \\
& =\int_{0}^{\infty} \ell(\hat{\theta}-\theta) \Pi\left(\mathrm{t}_{1}, \ldots, \mathrm{t}_{\mathrm{n}} ; \theta, \mathrm{p}\right) \mathrm{d} \theta \\
& =\mathrm{c} \hat{\theta}^{2}-2 \mathrm{c} \hat{\theta} \int_{0}^{\mathrm{n}} \frac{\left(\sum_{\mathrm{i}=1}^{\mathrm{n}} \mathrm{t}_{\mathrm{i}}^{\mathrm{p}}\right)^{\mathrm{n}}}{\theta^{\mathrm{n}+1}(\mathrm{n}-1) !} \exp \left(-\frac{\sum_{\mathrm{i}=1}^{\mathrm{n}} \mathrm{t}_{\mathrm{i}}^{\mathrm{p}}}{\theta}\right) \mathrm{d} \theta+\psi(\theta)
\end{aligned}
$$

Let $\frac{\partial \mathrm{R}(\hat{\theta}-\theta)}{\partial \hat{\theta}}=0$, then the Bayes estimator is:

$$
\hat{\theta}_{\mathrm{BJ}}=\frac{\left(\sum_{\mathrm{i}=1}^{\mathrm{n}} \mathrm{t}_{\mathrm{i}}^{\mathrm{p}}\right)^{\mathrm{n}}}{(\mathrm{n}-1) !} \int_{0}^{\infty} \theta^{-\mathrm{n}} \exp \left(\frac{\sum_{\mathrm{i}=1}^{\mathrm{n}} \mathrm{t}_{\mathrm{i}}^{\mathrm{p}}}{\theta}\right)
$$

the scale parameter for Jeffrey prior is:

$$
\hat{\theta}_{B J}=\frac{\sum_{i=1}^{n} t_{i}^{p}}{n-1}
$$

Extension of Jeffery Prior Information: The extension of Jeffrey prior is taking $\mathrm{g}(\theta) \propto\left[(\mathrm{I}(\theta)]^{\mathrm{c}}, \mathrm{c} \in \mathrm{R}^{+}\right.$ then $g(q)=k \frac{n^{c}}{\theta^{2 c}} k$ is a constant.
The same way above, we can find the Bayes estimator with extension of Jeffrey prior depend on conditional probability density function as following: The joint probability density function is given by:

$$
\begin{aligned}
H\left(t_{1}, \ldots, t_{n}, \theta, p\right) & =\prod_{i=1}^{n} f(t, \theta, p) g(\theta) \\
& =\frac{k^{c} p^{n}}{\theta^{2 c+n}} \exp \left((p-1) \sum_{i=1}^{n} \ln t_{i}-\frac{\sum_{i=1}^{n} t_{i}^{p}}{\theta}\right)
\end{aligned}
$$

The marginal probability density function of $\left(t_{1}, \ldots, t_{n}\right)$ is given by:

$$
\begin{aligned}
\mathrm{P}\left(\mathrm{t}_{1}, \ldots, \mathrm{t}_{\mathrm{n}}\right) & =\int \mathrm{H}\left(\mathrm{t}_{1}, \ldots . \mathrm{t}_{\mathrm{n}} ; \theta, \mathrm{p}\right) \mathrm{d} \theta \\
& =\frac{\mathrm{kn}^{\mathrm{c}} \mathrm{p}^{\mathrm{n}} \exp \left((\mathrm{p}-1) \sum_{\mathrm{i}=1}^{\mathrm{n}} \ln \mathrm{t}_{\mathrm{i}}\right)(2 \mathrm{c}+\mathrm{n}-2) !}{\left(\sum_{\mathrm{i}=1}^{\mathrm{n}} \mathrm{t}_{\mathrm{i}}^{\mathrm{p}}\right)^{2 \mathrm{c}+\mathrm{n}-1}}
\end{aligned}
$$

The conditional probability density function of $\theta$ given by:

$$
\begin{aligned}
\Pi\left(\mathrm{t}_{1}, \ldots, \mathrm{t}_{\mathrm{n}} ; \theta, \mathrm{p}\right) & =\frac{\mathrm{H}\left(\mathrm{t}_{1}, \ldots, \mathrm{t}_{\mathrm{n}} ; \theta, \mathrm{p}\right)}{\mathrm{p}\left(\mathrm{t}_{1}, \ldots, \mathrm{t}_{\mathrm{n}}\right)} \\
& =\frac{\left(\sum_{\mathrm{i}=1}^{\mathrm{n}} \mathrm{t}_{\mathrm{i}}^{\mathrm{p}}\right)^{2 \mathrm{c}+\mathrm{n}-1} \exp \left(-\frac{\sum_{\mathrm{i}=1}^{\mathrm{n}} \mathrm{t}_{\mathrm{i}}^{\mathrm{p}}}{\theta}\right)}{\theta^{2 \mathrm{c}+\mathrm{n}}(2 \mathrm{c}+\mathrm{n}-2) !}
\end{aligned}
$$

The Risk function:

$$
\begin{aligned}
& \mathrm{R}(\hat{\theta}-\theta)=\mathrm{E} \ell(\hat{\theta}-\theta) \\
& =\int_{0}^{\infty} \ell(\hat{\theta}-\theta) \Pi\left(\mathrm{t}_{1}, \ldots, \mathrm{t}_{\mathrm{n}} ; \theta, \mathrm{p}\right) \mathrm{d} \theta \\
& =\mathrm{c} \hat{\theta}^{2}-2 \mathrm{c} \hat{\theta} \int_{0}^{\mathrm{n}} \int_{\mathrm{i}=1}^{\mathrm{n}} \frac{\left.\sum_{\mathrm{i}}^{\mathrm{n}} \mathrm{t}_{\mathrm{i}}^{\mathrm{p}}\right)^{2 \mathrm{c}+\mathrm{n}-1} \exp \left(-\frac{\sum_{\mathrm{i}=1}^{\mathrm{n}} \mathrm{t}_{\mathrm{i}}^{\mathrm{p}}}{\theta}\right)}{\theta^{2 \mathrm{c}+\mathrm{n}}(2 \mathrm{c}+\mathrm{n}-2) !} \mathrm{d} \theta+\psi(\theta) \\
& \text { Let } \frac{\partial \mathrm{R}(\hat{\theta}-\theta)}{\partial \hat{\theta}}=0, \text { then the Bayes estimator is: } \\
& \hat{\theta}_{\mathrm{BE}}=\frac{\left(\sum_{\mathrm{i}=1}^{\mathrm{n}} \mathrm{t}_{\mathrm{i}}^{\mathrm{p}}\right)^{2 \mathrm{c}+\mathrm{n}-1}}{(2 \mathrm{c}+\mathrm{n}-2) !} \int_{0}^{\infty} \frac{1}{\theta^{2 \mathrm{c}+\mathrm{n}-1}} \exp \left(-\frac{\sum_{\mathrm{i}=1}^{\mathrm{n}} \mathrm{t}_{\mathrm{i}}^{\mathrm{p}}}{\theta}\right) \mathrm{d} \theta
\end{aligned}
$$




\section{J. Math. \& Stat., 6 (2): 100-104, 2010}

the scale parameter for extension Jeffrey prior is:

$$
\hat{\theta}_{\mathrm{BE}}=\frac{\sum_{\mathrm{i}=1}^{\mathrm{n}} \mathrm{t}_{\mathrm{i}}^{\mathrm{p}}}{2 \mathrm{c}+\mathrm{n}-2}
$$

\section{RESULTS}

In this simulation study, we have chosen $n=25$, 50, 100 to represent small moderate and large sample size, several values of parameter $\theta=0.5,1.5$ and $p=0.8,1.2$, two values of Jeffery extension $c=0.4,0.8$.

\begin{tabular}{|c|c|c|c|c|c|c|}
\hline Size & $\theta$ & $\mathrm{C}$ & $\mathrm{P}$ & MLE & Bayes & Extension \\
\hline \multirow[t]{8}{*}{25} & \multirow[t]{4}{*}{0.5} & \multirow[t]{2}{*}{0.4} & 0.8 & 0.0190 & 0.0243 & 0.0256 \\
\hline & & & 1.2 & 0.0118 & 0.0105 & 0.0103 \\
\hline & & \multirow[t]{2}{*}{0.8} & 0.8 & 0.0190 & 0.0243 & 0.0209 \\
\hline & & & 1.2 & 0.0118 & 0.0105 & 0.0112 \\
\hline & \multirow{4}{*}{1.5} & \multirow[t]{2}{*}{0.4} & 0.8 & 0.0908 & 0.0874 & 0.0876 \\
\hline & & & 1.2 & 0.1238 & 0.1549 & 0.1627 \\
\hline & & \multirow[t]{2}{*}{0.8} & 0.8 & 0.0908 & 0.0874 & 0.0887 \\
\hline & & & 1.2 & 0.1238 & 0.1549 & 0.1348 \\
\hline \multirow[t]{8}{*}{50} & \multirow[t]{4}{*}{0.5} & \multirow[t]{2}{*}{0.4} & 0.8 & 0.0122 & 0.0143 & 0.0148 \\
\hline & & & 1.2 & 0.0083 & 0.0074 & 0.0072 \\
\hline & & \multirow[t]{2}{*}{0.8} & 0.8 & 0.0122 & 0.0143 & 0.0130 \\
\hline & & & 1.2 & 0.0083 & 0.0074 & 0.0079 \\
\hline & \multirow{4}{*}{1.5} & \multirow[t]{2}{*}{0.4} & 0.8 & 0.0543 & 0.0500 & 0.0493 \\
\hline & & & 1.2 & 0.0702 & 0.0817 & 0.0844 \\
\hline & & \multirow[t]{2}{*}{0.8} & 0.8 & 0.0543 & 0.0500 & 0.0524 \\
\hline & & & 1.2 & 0.0702 & 0.0817 & 0.0745 \\
\hline \multirow[t]{8}{*}{100} & \multirow[t]{4}{*}{0.5} & \multirow[t]{2}{*}{0.4} & 0.8 & 0.0090 & 0.0100 & 0.0094 \\
\hline & & & 1.2 & 0.0062 & 0.0057 & 0.0056 \\
\hline & & \multirow[t]{2}{*}{0.8} & 0.8 & 0.0090 & 0.0100 & 0.0094 \\
\hline & & & 1.2 & 0.0062 & 0.0057 & 0.0060 \\
\hline & \multirow[t]{4}{*}{1.5} & \multirow[t]{2}{*}{0.4} & 0.8 & 0.0340 & 0.0313 & 0.0308 \\
\hline & & & 1.2 & 0.0442 & 0.0492 & 0.0503 \\
\hline & & \multirow[t]{2}{*}{0.8} & 0.8 & 0.0340 & 0.0313 & 0.0329 \\
\hline & & & 1.2 & 0.0442 & 0.0492 & 0.0461 \\
\hline
\end{tabular}

Table 2: MPE of estimated parameters of Weibull distribution

\begin{tabular}{|c|c|c|c|c|c|c|}
\hline Size & $\theta$ & $\mathrm{C}$ & $\mathrm{P}$ & MLE & Bayes & Extension \\
\hline \multirow[t]{8}{*}{25} & \multirow[t]{4}{*}{0.5} & \multirow[t]{2}{*}{0.4} & 0.8 & 0.2146 & 0.2449 & 0.2519 \\
\hline & & & 1.2 & 0.1806 & 0.1673 & 0.1653 \\
\hline & & \multirow[t]{2}{*}{0.8} & 0.8 & 0.2146 & 0.2449 & 0.2257 \\
\hline & & & 1.2 & 0.1806 & 0.1673 & 0.1748 \\
\hline & \multirow[t]{4}{*}{1.5} & \multirow[t]{2}{*}{0.4} & 0.8 & 0.1636 & 0.1588 & 0.1585 \\
\hline & & & 1.2 & 0.1827 & 0.2040 & 0.2091 \\
\hline & & \multirow[t]{2}{*}{0.8} & 0.8 & 0.1636 & 0.1588 & 0.1610 \\
\hline & & & 1.2 & 0.1827 & 0.2040 & 0.1904 \\
\hline \multirow[t]{8}{*}{50} & \multirow[t]{4}{*}{0.5} & \multirow[t]{2}{*}{0.4} & 0.8 & 0.1753 & 0.1916 & 0.1951 \\
\hline & & & 1.2 & 0.1539 & 0.1435 & 0.1416 \\
\hline & & \multirow[t]{2}{*}{0.8} & 0.8 & 0.1753 & 0.1916 & 0.1815 \\
\hline & & & 1.2 & 0.1539 & 0.1435 & 0.1495 \\
\hline & \multirow[t]{4}{*}{1.5} & \multirow[t]{2}{*}{0.4} & 0.8 & 0.1281 & 0.1218 & 0.1208 \\
\hline & & & 1.2 & 0.1386 & 0.1497 & 0.1522 \\
\hline & & \multirow[t]{2}{*}{0.8} & 0.8 & 0.1281 & 0.1218 & 0.1254 \\
\hline & & & 1.2 & 0.1386 & 0.1497 & 0.1428 \\
\hline \multirow[t]{8}{*}{100} & \multirow[t]{4}{*}{0.5} & \multirow[t]{2}{*}{0.4} & 0.8 & 0.1574 & 0.1673 & 0.1613 \\
\hline & & & 1.2 & 0.1383 & 0.1315 & 0.1302 \\
\hline & & \multirow[t]{2}{*}{0.8} & 0.8 & 0.1574 & 0.1673 & 0.1613 \\
\hline & & & 1.2 & 0.1383 & 0.1315 & 0.1356 \\
\hline & \multirow[t]{4}{*}{1.5} & \multirow[t]{2}{*}{0.4} & 0.8 & 0.1031 & 0.0981 & 0.0953 \\
\hline & & & 1.2 & 0.1097 & 0.1167 & 0.1213 \\
\hline & & \multirow[t]{2}{*}{0.8} & 0.8 & 0.1031 & 0.0981 & 0.1010 \\
\hline & & & 1.2 & 0.1097 & 0.1167 & 0.1124 \\
\hline
\end{tabular}

The number of replication used was $\mathrm{R}=1000$. The simulation program was written by using Matlab program. After the parameter was estimated, Mean Square Error (MSE) and Mean Percentage Error (MPE) were calculated to compare the methods of estimation, where:

$$
\operatorname{MSE}(\theta)=\frac{\sum_{i=1}^{1000}\left(\hat{\theta}_{i}-\theta\right)^{2}}{\mathrm{R}} \quad \operatorname{MPE}(\theta)=\frac{\sum_{i=1}^{1000} \frac{\left|\hat{\theta}_{\mathrm{i}}-\theta\right|}{\theta}}{\mathrm{R}}
$$

The results of the simulation study are summarized and tabulated in Table 1-2 for the MSE and the MPE of the three estimators for all sample size and $\theta$, p values respectively.

In each row of Table 1-2 we have three values of estimators that is the MLE estimator, Jeffrey prior and extension of Jeffrey prior. The best method is the method that gives the smallest value of (MSE) and (MPE).

\section{DISCUSSION}

In Table 1, when we compared parametric estimators of Weibull distribution in Maximum Likelihood (MLE) and Bayes using Jeffery prior and extension of Jeffery prior by Mean Square Error (MSE) we find the best estimator is Maximum Likelihood (MLE) by $50 \%$ but it is clear from the Table 1 when $\mathrm{c}=0.4$ the Maximum Likelihood (MLE) is equal to extension of Jeffrey prior and when $\mathrm{c}=0.8$ the maximum likelihood (MLE) is equal to Bayes using Jeffrey prior.

In Table 2, when we compared parametric estimators of Weibull distribution in Maximum Likelihood (MLE) and Bayes using Jeffery prior and extension of Jeffery prior by Mean Percentage Error (MPE) we find the best estimator is Maximum Likelihood (MLE) by $50 \%$ but it is clear from the Table 2 when c $=0.4$ the Maximum Likelihood (MLE) is equal to extension of Jeffrey prior and when $\mathrm{c}=0.8$ the Maximum Likelihood (MLE) is equal to Bayes using Jeffrey prior.

\section{CONCLUSION}

The estimated parameters of Weibull distribution obtained from the maximum likelihood estimation is the best compared to Bayes. Bayes using extension of Jeffrey prior gives better result than Bayes using Jeffrey prior. 
When the number of sample size increases the Mean Square Error (MSE) and mean percentage error (MPE) decrease in all cases.

\section{REFERENCES}

Cohen, A.C. and B. Whitten, 1982. Modified maximum likelihood and modified moment estimators for the three-parameter Weibull distribution. Commun. Stat. Theory Methods, 11: 2631-2656. DOI: 10.1080/03610928208828412

Hossain, A.M. and W.J. Zimmer, 2003. Comparison of estimation methods for Weibull parameters: Complete and censored samples. J. Stat. Comput. Simul,73:145153.DOI:10.1080/009496502100003 3486.

Kantar, Y.M. and B. Penoolu, 2008.A comparative study for the location and scale parameters of the weibull distribution with given shape parameter. Comput. Geosci., 34: 1900-1909. DOI: 10.1016/j.cageo.2008.04.004
Sinha, S. K. and J.A. Sloan, 1988. Bayes estimation of the parameters and reliability function of the 3parameter Weibull distribution. IEEE Trans. Reliab., $\quad 37$ : 364-356. 10.1016/j.jspi.2004.04.018

Soland, R.M., 1969. Bayesian analysis of the Weibull Process with unknown scale and shape parameters. IEEE Trans. Reliab. R., 18: 181-184. DOI: 10.1109/TR.1969.5216348

Soliman, A.A., A. H.Abd Ellah and K.S. Sultan, 2006. Comparison of estimates using record statistics from Weibull model: Bayesian and non-Bayesian approaches. Comput. Stat. Data Anal., 51: 20652077. DOI: 10.1016/j.csda.2005.12.020 